Revue d'histoire de l'enfance « irrégulière »

Le Temps de l'histoire

$4 \mid 2002$

Images de l'enfance et de la jeunesse "irrégulières »

\title{
Le rôle des équipements médiatiques dans l'esthétisation des souffrances et des professionnalités
}

Éric Lepointe et Vincent Meyer

\author{
(2) OpenEdition \\ Journals \\ Édition électronique \\ URL : http://journals.openedition.org/rhei/59 \\ DOI : $10.4000 /$ rhei.59 \\ ISBN : 978-2-7535-1642-7 \\ ISSN : $1777-540 \mathrm{X}$ \\ Éditeur \\ Presses universitaires de Rennes \\ Édition imprimée \\ Date de publication : 15 novembre 2002 \\ Pagination : 169-199 \\ ISSN : 1287-2431
}

Référence électronique

Éric Lepointe et Vincent Meyer, « Le rôle des équipements médiatiques dans l'esthétisation des souffrances et des professionnalités », Revue d'histoire de l'enfance «irrégulière » [En ligne], 4 | 2002, mis en ligne le 18 mai 2007, consulté le 04 décembre 2020. URL : http://journals.openedition.org/rhei/59 ; DOI : https://doi.org/10.4000/rhei.59 


\section{Le rôle des équipements médiatiques dans l'esthétisation des souffrances et des professionnalités}

Éric

Lepointe $^{(1)}$

Vincent

Meyer $^{(2)}$

(1) Assistant de service social à la Protection judiciaire de la jeunesse. Enquêteur de personnalité auprès de la cour d'assises des Vosges.

(2) Sociologue, maître de conférences en sciences de l'information et de la communication à l'IUT "A" NancyVerdun. Chercheur au Centre de recherche sur les médias (groupe Praxis) et chercheur associé à l'équipe de recherche d'anthropologie et de sociologie de l'expertise, université de Metz.

(3) Vincent Meyer, Interventions sociales et médias entre professionnalisation et déprofession-

Éric Lepointe et Vincent Meyer / p. 167 à 199 
nalisation, thèse pour le doctorat de sociologie, université de Metz, 1998. Une version remaniée est à paraître aux éditions L'Harmattan dans la collection "Logiques sociales".

(4) L'équipement est pris au sens où l'emploie J.Y. Trépos, comme " un dispositif liant personnes, choses et actions selon une certaine loi, dans lequel les partenaires acceptent d'investir et à propos desquels ils s'accordent.

L'acception du terme est donc large et va, en toute rigueur, de l'équipement objectivé (bâtiment, machine, livre, film, algorithme...) à l'équipement incorporé (savoir ou savoir-faire, disposition éthique...). » Jean-Yves Trépos, Sociologie de l'expertise, Paris, Presses universitaires de France, 1996, p. 49-50. image et en scène de la souffrance contemporaine ; celle-ci ne se présente ni se reconnaît spontanément ; elle doit être esthétisée et transmise, notamment par les médias. De facto, ces processus sont éminemment instables et la réalité de la souffrance n'est jamais définie une fois pour toutes. C'est de ces processus qu'il sera plus particulièrement question ici.

Ce texte s'ancre également dans un travail d'observation participante périphérique et de recueil de données journalistiques réalisés par un professionnel de terrain au moment d'une affaire de maltraitance d'enfant, jugée du 28 septembre au 1er octobre 1999 à la cour d'assises des Vosges. Aussi, il nous faut de suite avertir le lecteur. Il ne s'agit pas, dans ce texte, de se livrer à une montée en généralité à partir d'une situation de maltraitance et de son traitement médiatique, mais bien de soumettre ces derniers - dans une perspective constructiviste et avec la prudence méthodologique qui s'impose - à une modélisation construite au cours de la recherche évoquée supra. Ainsi, on n’ignore pas que les documents et témoignages sur lesquels nous avons travaillé sont aussi des vulgates, des traductions et des construits sociaux particuliers et instables. De même, nous contribuons par ce texte et par les positions que nous occupons dans nos champs respectifs à la construction et la validation de la mémoire des événements et à la légitimation de certains équipements. Mais ceci est certainement - selon une formule maintenant consacrée - un risque acceptable. Les souffrances et l'épisode juridico-médiatique de Johnny, "le petit martyr des Vosges", (5) ne sont, certes pas, uniques et exemplaires. Ceci posé, ils n'ont pas fait l'objet d'un traitement aléatoire ou simplement événementiel et permettent, selon nous, de saisir et d'illustrer plus finement l'évolution de ce qui semble aujourd'hui "aller de soi" dans le tramage entre le traitement de certains problèmes de société et leur vision médiatique.

On se propose donc de partir de la mise au jour - forcément incomplète - d'une souffrance et "de l'agitation et de la déferlante médiatiques" dont elle a fait l'objet. De déconstruire et d'analyser ensuite les différents équipements nécessaires aujourd'hui à la construction d'une esthétique de la souffrance et, enfin, à celle des professionnalités des agents du social. On reviendra, dans la conclusion, sur les différentes 
coconstructions en cours et sur l'avènement d'un champ appelé "sociomédiatique".

\section{Il était une fois... un enfant martyr, des adultes maltraitants et/ou consentants, des médias coconstruisant les événements...}

\section{1. (Re)prenons l'histoire}

Joël est artisan menuisier. Pendant l'été 1996, il a remplacé les fenêtres d'une ferme de Ménil-en-Xantois dans les Vosges. Comme il tarde à être payé, il y retourne courant octobre pour réclamer son dû, lorsque, comme le publiera Femmes actuelles (18 août 1997), "un petit garçon s'est tourné vers lui, le regard suppliant. Il a vu son ail à moitié fermé, il a senti la peur qui habitait l'enfant. Alors son cour s'est mis à battre plus vite en reconnaissant l'horreur que lui-même avait vécue dans son enfance; lui aussi a été maltraité par une marâtre. Alors Joël lui fait un clin d'oil, puis se précipite à la gendarmerie de Mirecourt."

Les parents de Johnny le petit garçon, Sandra et René, sont rapidement placés en garde à vue, puis mis en examen pour tortures et actes de barbarie de manière habituelle sur mineur de quinze ans. Johnny, placé une première fois par le juge des enfants, leur avait été remis un an auparavant, une éducatrice étant chargée d'une mesure éducative en milieu ouvert (c'est-à-dire à domicile). Notons que cette éducatrice avait perdu leur trace depuis deux mois, puisqu'ils avaient quitté leur appartement précédent sans laisser d'adresse et étaient venus s'installer à Ménil. Dans cette maison vivaient cinq autres personnes, plus ou moins apparentées. Deux seront mises en examen pour les mêmes chefs, les autres l'étant pour non-dénonciation de crime. Le substitut du procureur déclare alors : "Si cette affaire n'était pas sortie rapidement, il y a de fortes chances que l'enfant serait mort à l'heure actuelle. » Interrogés par les gendarmes, les prévenus, qui parlent beaucoup, semblent ne rien cacher du calvaire qu'ils ont fait subir à cet enfant de quatre ans, hospitalisé à Nancy.

Commence le week-end prolongé de la Toussaint 1996. La voisine d'en face se souvient : "Quand j'ai ouvert mes volets, il y avait plein de journalistes, les télévisions, les radios. » Michel, qui n’a pas été incarcéré, leur 
ouvre la maison, montre l'improbable matelas où dormait Johnny lorsqu'il n'était pas avec les cochons et raconte, lui aussi. Paris Match (14 novembre 1996), qui publie la photo du baptême de Johnny, écrit : "Souvent au cours de son sommeil, on le réveille pour le battre. Au matin, puni, il assiste sans y participer au petit déjeuner des adultes. Les hommes partis travailler, les femmes se déchaînent. Assommé de coups, Johnny prend l'babitude de se réfugier dans un recoin de la cuisine entre la porte et le mur. Frappé à coups de casseroles et de poêle à frire, piqué avec des fourchettes, insulté sous le moindre prétexte, se nourrissant des restes que les adultes veulent bien lui laisser, Johnny expie des journées durant, un crime qu'il ignore : celui d'être né de rapports interdits."

Ainsi, se penchant aussi sur les bourreaux, on évoque notamment l'enfance bafouée de Sandra la mère, violée dès ses quatorze ans par Claude, son beau-père qui est aussi son oncle. L'auteur de cet article de Paris Match, qui est également journaliste à la Liberté de l'Est, y affirme, le 5 novembre, que Johnny est né de ces relations imposées. Or ce n'est que pure allégation. Ce même quotidien, le lendemain, titre : "Vague d'émotion autour de Johnny. Le cas du petit Johnny a ému toute la France et même certains pays limitrophes comme la Belgique. Des centaines d'appels téléphoniques et de courriers affluent tant à Nancy, Epinal ou Golbey où réside l'enfant depuis le 28 octobre."

Ensuite, ce sera le silence dans les médias. L'enquête est simple à certains égards : les accusés (à l'exception peut-être de Sandra) avouent tout, ne se renvoient pas la balle ; il n'y a pas de zones d'ombre quant aux faits, donc ni mystère, ni intrigue, ni suspense. Toutefois la presse écrite revient parfois sur Joël, son épouse et leurs cinq enfants, qui s'épuisent en démarches diverses auprès des services sociaux en vue d'adopter Johnny, sinon devenir sa famille d'accueil ; en vain.

Au début de l'année 1998, un journaliste et un des auteurs de ces lignes bavardent dans les couloirs du palais de justice d'Épinal. Le premier, troublé, doute. Il a eu vent que le certificat médical établi après examen du garçon, ne fixe l'incapacité totale temporaire qu'à huit jours. Qu'en est-il alors de la réalité de la souffrance ? Son interlocuteur, qui connait le dossier, lui répond que seules les conséquences d'un des aspects de la violence sont ici mesurées, les marques et les séquelles 
concrètes sur le corps. L'essentiel des dommages est sans doute ailleurs. Ainsi, à quatre ans, percevoir son impuissance absolue face à un adversaire immensément plus puissant, cruel et imprévisible, éprouver le sentiment non pas d'avoir fait une faute, mais d'en être une, être confronté à la perspective de sa propre mort... ne peut pas ne pas laisser de traces. Le journaliste attentif ne commente pas!

Et puis arrive le procès d'assises, qui se déroule à Épinal du mardi 28 septembre au vendredi 1er octobre 1999. Ils sont tous là : Sandra, René, Claude, Marie-France, Murielle, Raphaël et Michel... TF1, France 2 et France 3, M6, Canal +, RTL, Europe 1, Le Monde, Libération, Le Provençal, L'Est républicain, l'AFP... Flashs et projecteurs saturent les visages des accusés dans leur box. Sandra proteste. Photographes et cameramen doivent se retirer, l'audience commence.

TF1 avait préalablement prié le procureur de bien vouloir mettre une salle à disposition de son équipe. Le procureur ne lui a pas répondu. TF1, qui avait réussi à connaître le nom et l'adresse de la famille d'accueil de Johnny, avait insisté auprès du conseil général pour être autorisé à filmer le garçon dans son village. Estimant, tout compte fait, qu'il valait mieux consentir et encadrer que refuser et être dépassé, le conseil général autorise. Une exclusivité pour le journal de $20 \mathrm{~h}$.

Au cours de l'audience, enquêteurs de personnalité, experts psychologues et psychiatres se suivent à la barre pour dresser le portrait des sept accusés ; portrait où il est question d'inceste, de sadisme, d'absence totale de sentiment de culpabilité, de pauvreté culturelle et intellectuelle, voire de débilité... On regrette de n'avoir pu évaluer la dynamique de cette "borde" coalisée contre un. Cette première audience sera levée à $18 \mathrm{~h} 30$, mais les équipes de télévision sont déjà parties depuis 16h, laissant boîtes de jus de fruits, mégots et journaux froissés joncher le sol du hall.

Le mercredi matin, sera poursuivi l'examen de personnalité. Équipes de télévision et de radio s'emparent des experts dès leur sortie. Une psychologue est cernée. Elle doit parler. Elle parle. Plus tard, revenue dans la salle, elle s'en veut, elle leur en veut : «Ils m'ont eue, piégée ! C'est pas vrai ! J'avais pas imaginé, j’allais aux toilettes !» Elle ressort, prépa- 
rée et déterminée cette fois, et leur interdit de diffuser ces images. Elle semble avoir été entendue.

Le lendemain, la Liberté de l'Est publie deux photos de journalistes, cameramen et reporters au travail et titre: "Johnny : déferlante médiatique aux marches du palais. Le tribunal connaît une agitation qui n'a pas été sans rappeler l'affaire du petit Grégory." De son côté, Le Monde donne des précisions : "Au deuxième jour, les jurés se sont passé certains instruments du crime. Des casseroles en aluminium, une sauteuse, une poêle, une passoire en plastique [...] les bosses sur le métal proviennent des coups portés durant quatre mois à Johnny." Et Libération de reprendre les propos de Marie-France: "C'est devenu machinal, ça devait me défouler et ça créait une bonne ambiance à la maison; on l'insultait, ça faisait rire tout le monde. On avait envie de le tuer, sans ça on aurait pas tapé autant."

L'éducatrice chargée de la mesure éducative arrive à la barre. Durant une heure environ, son travail éducatif auprès de Johnny et de sa famille est comme autopsié. Dans le rôle des "médecins-légistes » : l'avocat de Sandra et les deux avocates d'Enfance et partage et Enfance majuscule. La veille, cette dernière s'était confiée sur France 3 : "Il s'agira de dénoncer les carences des services sociaux et dire tout le bien de la personne qui est intervenue et a sauvé l'enfant." L'éducatrice fait front. Elle dit ne pas avoir constaté de maltraitance, puis, convenant avec une des avocates de la partie civile que les signaux d'alarme étaient au rouge, elle poursuit : "Je ne suis que des familles dont les signaux sont au rouge." Puis, elle détaillera les démarches multiples qu'elle a vainement effectuées pour retrouver la trace du garçon et de ses parents, parmi lesquelles une recherche d'adresse auprès de la caisse d'allocations familiales et une sollicitation auprès de la grand-mère maternelle du garçon.

Plus tard, une rumeur se répand au tribunal : peut-être Johnny sera présent demain. Là, dans la salle, sous nos yeux. Son avocate l'a souhaité, un expert psychologue l'a préconisé faisant valoir les vertus thérapeutiques de sa venue au procès.

La salle d'audience a toujours été comble. Ce jeudi, elle l'est deux fois plus. La Liberté de l'Est s'interroge : "Viendra, viendra pas ? Voilà la question qui hantait les allées du palais de justice d'Épinal durant la journée de jeudi. 
[...] Et, à 16h20, à la reprise des débats, un petit bonhomme aux cheveux blonds est entré dans la salle pour venir se mettre sur les genoux de son assistante sociale. Johnny, habillé d'un jogging bleu et d'un sweat-shirt blanc, a serré son nounours contre sa poitrine." L’Est républicain précise en substance que "le public se hausse sur la pointe des pieds pour l'apercevoir". L'assistante sociale nous confiera plus tard : "À côté de moi, une journaliste commençait à s'agiter, à demander si le garçon était bien Johnny. Quand il a entendu ça, il a voulu partir, on est parti. » Il n'aura pas été là plus de cinq minutes. Le chroniqueur judiciaire de RTL, très remonté contre l'avocate à l'initiative de ce qu'il appelle une mascarade, travaille son texte : "Au $18 \mathrm{~h}$, je vais lui dire ce que j'en pense ». Promesse tenue. La presse, d'une manière générale, exprimera sa perplexité quant à la pertinence de la présence de Johnny.

À une interruption de séance, le procureur raconte que son fax reçoit message sur message de toute la France, lui réclamant des peines exemplaires. Soucieux de rester à distance de la voxpopuli, il sera modéré dans ses réquisitions. Sur TF1, il dira d'ailleurs : "La justice n'est pas le bras armé de l'opinion publique."

Vendredi en fin d'après-midi, la cour et les jurés se retirent pour délibérer, tandis que la SFP et TF1 déploient leurs paraboles devant le tribunal, barrant même une rue, pour annoncer le verdict en direct au journal télévisé. Mais les heures passent, la soirée avance. Les journalistes regardent nerveusement leur montre, attendent la clôture des travaux... Le Monde du lendemain : "Chaque jour, la foule n'avait cessé de grossir. Vendredi ler octobre, elle déborde, dans les travées, plus loin au dehors de la cour d'assises des Vosges. Il n'y a rien à voir, qu'une punition à entendre. Certains, pour ne pas perdre leur place, ont attendu assis, tout le temps des six beures de délibéré. Chaleur, bruit, ambiance d'hallali. Plusieurs sont venus en famille, avec des enfants. D’autres, dans le prétoire, ont amené leur chien. Alors les accents vosgiens remuent des histoires de famille, d'éducation, d'enfants. On parle aussi de justice, des peines qu'on voudrait voir infliger."

Un peu avant $22 \mathrm{~h}$, les accusés, la cour et les jurés reprennent place. Le verdict est prononcé tandis que photographes et cameramen, depuis le hall, perchés sur des escabeaux, mitraillent et filment les accusés. Le pré- 
(6) Brièvement, dans les deux cas, mon travail a consisté à présenter le parcours biographique des accusés (éventuellement des victimes) et à faire leur portrait, en quelque sorte. sident s'interroge un instant : "N'est-ce pas interdit de filmer et d'enregistrer les débats ? " Mais il y a cette malencontreuse porte en verre ! Il termine sa lecture du prononcé des peines avant de lever la séance. Les journalistes appellent leur rédaction. Télévisions et radios interviewent une dernière fois, tandis que France Info annonce déjà la nouvelle.

Arrivent les fêtes de Noël, qui verront les services sociaux du département submergés de peluches, envoyées de partout, à offrir à Johnny. Et, pour la session d'assises suivante, on a fait installer un store sur la porte vitrée. Le rideau est-il définitivement tombé ?

\subsection{Brèves réflexions d'un professionnel}

Cette affaire m'amène à réfléchir à deux questions. Pourquoi a-t-elle mobilisé à ce point les médias ? La pression médiatique et la présence d'une foule nombreuse aux audiences ont-elles pu modifier le déroulement du procès et peser sur le verdict?

\subsubsection{La mobilisation des médias}

En dix ans (la période couvre les années quatre vingt-dix), j'ai participé à environ soixante-dix procès criminels en tant qu'enquêteur de personnalité (pour les majeurs) ou assistant social de la Protection judiciaire de la jeunesse (pour les mineurs). ${ }^{(6)}$ Parmi toutes ces affaires, celle-ci, manifestement, se distingue par son traitement médiatique. Alors pourquoi celle-ci ?

$\mathrm{Si}$ on postule que le sordide excite la curiosité (malsaine ajoute-t-on comme pour s'en démarquer) et que le sort des enfants victimes de maltraitance produit généralement une intense émotion, j’observe que bien d'autres affaires la dépassèrent dans le sordide, tandis que d'autres concernant aussi d'émouvants enfants-victimes n'ont guère généré un tel traitement médiatique. Si on postule que l'énigme judiciaire éveille l'intérêt, constatons qu'il n'y en eu aucune ici. De même, cette affaire n'est pas venue interroger notre conscience morale, ni nourrir un débat de société comme l'aurait suscité un cas d'euthanasie par exemple. Cette affaire ne me paraît nullement exemplaire, ni exceptionnelle, ni un opportun support pédagogique pour l'opinion publique... 
Alors pourquoi cette affaire est-elle sortie et pas une autre ? Par quelle alchimie ? À partir de quels ingrédients les directeurs de rédaction ont-ils simultanément décidé d'envoyer leur équipe de reporters à peine l'affaire ébruitée ? Dois-je me résoudre à penser comme un collègue : "C'est l'histoire des cochons qui a tout déclenché. Quand Johnny, puni, était envoyé avec eux. Tu vois le contraste entre la modernité à l'aube du xxième siècle et ces relents de Moyen Âge ? "Oui, je vois. Pourtant cet argument, sans doute pertinent en soi mais trop univoque, ne suffit pas, me semble-t-il, pour répondre à la question de manière satisfaisante. En effet, j'ai dans mes dossiers bien d'autres relents de Moyen Âge que les projecteurs n’ont pas éclairés.

\subsubsection{Pression médiatique et déroulement du procès}

Pris dans cette "déferlante médiatique", je n'ai pas le sentiment que la cour, le représentant du ministère public, les avocats ou les experts aient rempli leur mission différemment. De même, les accusés ne m’ont paru ni paralysés, ni portés à se mettre en scène. Par contre, je voudrais m'attarder sur Johnny. Pour ma part, je défends l'idée que la présence de la victime - fût-elle très jeune - à l'audience présente une vertu thérapeutique, pour peu qu'un certain nombre de conditions soient remplies, parmi lesquelles une sérénité à l'audience et surtout un certain degré d'intimité, introuvables dans un contexte de pression médiatique.

Généralement, le public des procès d'assises se réduit à quelques observateurs isolés, attentifs et fort discrets. En de telles circonstances, sa participation au procès eut été acceptable. Or, ici, une fois entré dans cette arène, en quelque sorte, Johnny fut soit objet de curiosité, soit passager indésirable pour ceux qui estimaient qu'il n'avait rien à faire là. Si mal considéré, ${ }^{(7)}$ il ne nous étonna pas lorsqu'il quitta la salle au bout de cinq minutes.

Ainsi, ce contexte particulier a participé à son éviction d'une "scène" où l'on pouvait estimer qu'il avait toute sa place. On pourrait aussi rétorquer qu'un procès d'assises est fait par des adultes pour des adultes. Mais rien n'empêchait de prévoir une séquence à huis clos au cours de laquelle le président, s'adressant au garçon en des termes adaptés à son
(7) En dépit d'un long et minutieux travail de préparation des services sociaux qui l'avaient en charge et de son avocate. 
(8) Luc Boltanski, La souffrance à distance, Paris, Métaillé, 1993.

(9) Patrick Champagne, "La vision médiatique”, in Pierre Bourdieu, [dir.], La misère du monde, Paris, Seuil, 1993, p. 72. âge, lui aurait expliqué ce qui se jouait là, que les adultes dans le box avaient commis des choses très graves, qu'il revenait à ce tribunal de les punir, enfin qu'il n'avait rien fait de mal. À propos du verdict, les observateurs, unanimes, se sont étonnés de constater que les peines, qualifiées de lourdes, dépassaient généralement les réquisitions de l'avocat général. N'est-ce pas le résultat d'un climat invitant à la sévérité et que le journaliste du Monde comparaît à une ambiance d'hallali ?

\section{L'esthétisation d'une souffrance}

L'illustration du cas de Johnny nous permet de mieux comprendre les étapes du processus de mise en scène et de mise en image de sa souffrance. Nous avons, d'une part, la mise au jour puis l'exposition auprès d'un public de la souffrance physique quotidienne d'un jeune enfant et, de l'autre, un intense traitement médiatique du procès de ses parents, ce traitement médiatique reprenant et détaillant les équipements par lesquels cette souffrance doit être éprouvée et reconnue de tous. Simultanément, les médias mettent en œuvre une expertise participante et se réclament d'une forme de participation civique qui, souvent, interroge l'efficacité voire l'utilité des dispositifs préexistants (dans ce cas, les services sociaux). Par ailleurs, ce processus de mise en scène et en image n'est pas sans incidences sur la construction des opinions dans l'espace public ; il participe aussi de ce que Luc Boltanski appelle « la crise de la pitié ", qui amène le " sollicité " à s'interroger sur la " réalité du malheur » et de la souffrance d'autrui et sur le "réalisme de l'action » entreprise pour y répondre. ${ }^{(8)}$ De même, on peut rejoindre Patrick Champagne, pour qui un événement médiatisé " peut fonctionner comme une sorte de test projectif auprès des différents acteurs sociaux qu'ils [les médias] interrogent, chacun pouvant y voir la confirmation de ce qu'il pense depuis longtemps ". ${ }^{(9)}$ L'exposition dans l'espace public comme le traitement médiatique se caractérisent donc surtout par une description et une identification - dans des temporalités et des lieux différents - de personnes, d'instruments et d'événements par le biais d'objets communs et surtout techniques. Ces derniers ont la particularité de ne pas pouvoir être maîtrisés de tout le monde. 
On soutient, dans notre modélisation, que c'est l'agrégation de cette exposition et de ce traitement qui produit l'esthétisation. Ainsi, "esthétiser" est d'abord à comprendre au sens d'une perception intellectualisée, équipée par des personnes, des événements, des objets et des lieux communs et techniques. Il faut préciser que ce sont les objets et les lieux techniques qui servent à traduire - notamment par l'image, le son et le texte - les différents équipements de la souffrance engendrés par la situation de maltraitance de l'enfant. Mais "esthétiser" une souffrance (comme d'ailleurs, mais nous y reviendrons, une professionnalité), c'est aussi la soustraire durablement aux impératifs de la justification et la valider aux yeux de différents spectateurs. Cette esthétisation doit donc produire un effet public autant qu'un effet sur les publics. Elle permet également une nouvelle orientation de l'action et une (re)codification du rapport à autrui souffrant (notamment en ce qui concerne la dénonciation, l'indignation et/ou la compassion face à cette souffrance). En reprenant l'analyse de Luc Boltanski, ${ }^{(10)}$ les médias dénoncent et peignent - mais aussi dé-finissent - cette souffrance et permettent aux spectateurs de sympathiser avec un malheureux (mais aussi avec ses bienfaiteurs, voire dans certains cas... avec ses persécuteurs), tout en les affranchissant de certains investissements ou contraintes liés à l'action directe. Tout ceci "prend" d'autant mieux que les différents publics ont une perception des souffrances contemporaines sans croyances évaluatives définitivement stabilisées. Dans certains phénomènes (par exemple dans les souffrances liées à l'exclusion sociale), les sceptiques sont plus nombreux que les convertis.

Pour affiner, puis élargir notre analyse, on se propose maintenant de détailler les différents équipements mobilisés dans la chaîne des traductions de la souffrance de Johnny; ensuite, d'évoquer d'autres équipements d'esthétisation et de montrer comme ils prennent place dans un nouveau militantisme de cause, inséparable d'un engagement médiatique et médiatisé et preuve de son utilité publique ou sociale.

\subsection{Johnny : traduire et équiper l'inqualifiable !}

Les vulgates reprises pour évoquer cette affaire sont très significatives des différentes traductions opérées par les observateurs experts et pro-

(10) Luc Boltanski, op. cit., 1993. 
(11) Bruno Latour,

La science en action, Paris, La Découverte, 1995, p. 202.

(12) Ibid., p. 154. fanes. Tout se passe comme si : « Pour Johnny, ça a pris, parce que c'était un enfant, parce que c'était les Vosges, parce que quelqu'un, par hasard, a découvert son calvaire... » Pour mieux saisir ces vulgates, il faut revenir sur cette description et identification - dans des temporalités et des lieux différents - des personnes, des objets et des événements. Une précision s'impose ici. Cette partie n'échappe pas à une construction stylistique toujours critiquable : l'énumération. Le lecteur doit avoir à l'esprit que cette énumération des actants (expression proposée par Bruno Latour pour désigner les humains et les non-humains) ${ }^{(11)}$ ne peut pas viser l'exhaustivité et qu'il s'agit, là aussi, d'une (re)construction ad hoc participant de cette esthétisation.

\subsubsection{Les personnes}

$\mathrm{Si}$ au centre de la "déferlante médiatique", objet de la "pression médiatique" et d'un "voyeurisme insupportable", se trouve un enfant maltraité, Johnny est celui qui, in fine, physiquement, apparaît le moins. Il est pourtant placé in medias res (au milieu des choses) et existe surtout, pour reprendre une formule de Bruno Latour, "dans le monde de papier des articles [de la presse] » et aux côtés d'une série "d'acteurs sémiotiques présentés dans le texte " et plus ou moins présents dans les différents lieux d'exposition (la ferme, le palais de justice....). .12 $^{(2)}$ Parmi ces acteurs sémiotiques, on retrouve d'abord "la horde de Ménil", décrite, dans un article de presse, comme dans un jeu des sept familles: "S qui éduque par les coups ; $R G$, le père, qui frappait fort ; $M-F C$, la fermière acariâtre, $M T$ qui avait la main leste; $M C$, le fermier, qui ne disait rien; $C V$, le papa-oncle de $S ; R T$, fils de $M-F$." Après le procès, Libération publiera encore une double page sur la personnalité des quatre auteurs principaux. Viennent ensuite : les professionnels de la justice, dont les déclarations et commentaires vont être partiellement publiés ; ceux du social, "sur la sellette"; enfin, ceux des médias (voir point 3.2.) avec, au premier plan, les journalistes présents à Épinal, "prêts à saisir tous les mouvements et la moindre déclaration lors des suspensions de séances". D’autres, plus extérieurs, sont encore convoqués dans les journaux locaux, à l'instar de ce patron d'un café spinalien, de cette boulangère-pâtissière présentée comme une "observatrice de choix". 
Enfin, la foule "des curieux", présente lors des différentes séances, mais plus nombreuse au moment de la venue de l'enfant à l'audience ("près de 300 personnes"). Ainsi, "la venue annoncée de Jobnny a suscité la curiosité d'un public à l'affût d'une émotion équivoque : la souffrance d'un enfant comme spectacle." À cette foule physiquement présente, il convient d'adjoindre celle, non chiffrable, des spectateurs à distance ou distanciés, dont la présence se manifeste à travers différents témoignages (appels téléphoniques, lettres, cadeaux...), eux-mêmes relayés par différents dispositifs techniques (voir infra). Il s'agit notamment là d'un des effets publics de l'esthétisation, qui consiste à se laisser prendre et étourdir par la pression médiatique et de s'élancer du côté où résonne l'éloge d'une souffrance médiatiquement constituée.

\subsubsection{Les objets et lieux communs ou techniques}

L'importance et l'histoire des objets et des lieux communs et techniques sont souvent négligées dans les analyses savantes de la souffrance, lorsqu'elle est liée à des phénomènes dits sociaux. Sans doute, pour Johnny, les épreuves comme les preuves sont aussi dé-finies par des objets et des lieux. L'esthétisation implique donc d'importer des objets communs et d'avoir recours à des dispositifs non-humains souvent complexes pour les rendre visibles. Dans le cas de Johnny, et toujours dans une perspective latourienne, on peut même avancer qu'ils sont déterminants pour remonter des faits accomplis à l'accomplissement des faits et surtout pour les faire "tenir ensemble", même temporairement.

Le répertoire des objets et des lieux communs va, en toute rigueur : de "la soue à cochon", du "placard" où l'enfant était enfermé, du "tonneau d'eau froide", du "champ d'orties" dans lequel il a été jeté dans cette "ferme" des "Vosges" (notons que les auteurs ne sont pas des exploitants agricoles), au "matelas mousse" sur lequel il passait ses nuits "à même le sol (ou avec les cochons) sans couverture, ni drap et avec le ventre vide", en passant par les ustensiles de cuisine, les "casserole ; passoire ; louche ; fourchette" (présentés à l'audience), du "manche du martinet qui n'avait plus de lanière" utilisés pour lui asséner des coups. Ce répertoire comprend aussi les différentes photos parues dans les presses régionale et nationale et dans différents 
magazines (par exemple, la mère avec le bébé le jour du baptême à côté d'un prêtre, à l'audience dans le box des accusés ; les autres auteurs, et plus particulièrement une tante, "la marâtre [...] au langage ordurier").

Du côté des objets techniques, on peut sérier l'important dispositif technique aux "marches du palais", notamment celui des nombreuses télévisions et autres organes de presse, comme les objectifs des caméras, les micros, les paraboles, etc., tous indispensables dans la chaîne des premières traductions. Mais aussi les téléphones au centre hospitalier universitaire, par lesquels transitent les demandes d'information au moment de l'hospitalisation de l'enfant. De même, le fax du palais de justice transmettant les différentes traductions profanes de ce que doit être un acte de justice et/ou une peine exemplaire.

\subsection{3. "Faire l'événement"}

Dans cette affaire, "faire l'événement", c'est d'abord dé-finir les différentes épreuves (physiques et psychologiques) par et avec des personnes et des objets pendant un "procès [proclamé] unique pour des actes de maltraitance exceptionnels de gravité sur fond de misère morale et matérielle" ; c'est ensuite (re)lier ces mêmes épreuves avec une "cause indiscutable". Celleci concerne un public-cible nettement délimité et apparait dans l'espace public comme inconditionnelle (l'engagement et/ou l'action ne sauraient être soumis à condition; il n'y a plus de doute de l'effort consenti ou à consentir) et stabilisée au niveau des rhétoriques de légitimation (il n'y a, en principe, plus personne à convaincre de l'existence du malheur et de la grandeur, de la justesse et de l'utilité de la cause). Dans le cas de Johnny, c'est, bien évidemment, la cause de l'enfance maltraitée qui est analysée, et les différentes épreuves qui font l'événement :

- servent à nourrir les débats et controverses des experts ; ainsi, au moment du procès, Le Monde a posé la question de l'utilité de la présence de Johnny à l'audience en convoquant des représentants-experts de la sphère "psy", mais aussi le président de l'association des juges des enfants ; ces derniers, pour répondre à l'événement, ont donné différentes traductions à destination du grand public de cette épreuve inattendue ;

- sont autant de preuves qui confirment et valident la justesse d'un 
engagement dans cette cause et la grandeur de ceux et celles qui la soutiennent.

L'événement est aussi (re)lié à d'autres plus anciens, en particulier à l'affaire hypermédiatisée du petit Grégory, présentée comme "un autre grand feuilleton [...] qui, en son temps, mobilisa pendant des mois et des mois la France toute entière". Il faut ajouter ici, comme cela a été évoqué dans la première partie, que toutes les affaires de maltraitance ne font pas "événement", ne connaissent pas - mais c'est la même chose - les mêmes engouement et traitement médiatique et ne font pas l'objet des mêmes mises en équivalence dans l'espace public.

On l'aura compris, cette esthétisation s'élabore non seulement lors d'événements ponctuels comme les gardes à vue, les mises en examen ou les procès..., mais se donne aussi à voir en différé dans la presse écrite, dans les journaux et magazines télévisés. Ceux-ci vont non seulement diffuser l'événement, mais aussi le "grandir" et l'"étendre", en traitant par exemple de la prise en charge par l'administration judiciaire des mineurs en danger, en reprenant des trajectoires ou des histoires de vie (mineurs battus ou martyrisés, mineurs délinquants ou violents...). Là encore des experts sont convoqués parfois, aux côtés d'anciennes victimes ; chacun donne sa traduction de la souffrance et des différentes "toiles de fond" qui lui donnent corps (alcoolisme, inceste, précarité de l'emploi, perte des repères familiaux, vulnérabilité, problèmes psychologiques, etc.). Ce faisant, on introduit toutefois une nouvelle échelle de mesure, un nouvel espace de justification de la cause comme de l'action auprès des différentes populations "en cause" et donc de nouvelles épreuves professionnelles pour ceux qui en ont la charge. C'est notamment pour cette raison que l'esthétisation prend également corps (pour être retravaillée) dans la presse spécialisée du champ social, à l'instar de Marceline Gabel, secrétaire permanente de la grande cause, qui évoque, dans le cas de Johnny, " un déferlement d'articles ou d'émissions redondantes [...] exhibant inlassablement les mêmes images de cet alignement pitoyable des accusés. " Elle parle encore d'une "véritable désinformation qui permettait d'abord de mettre en cause les services de Protection de l'enfant ", notamment pour ce qui concerne la durée des mauvais traitements infligés à l'enfant. ${ }^{(13)}$
(13) Marceline Gabel, "David, Laetitia, Johnny et les autres", Journal $d u$ droit des jeunes, $n^{\circ} 190$, 1999, p. 6. 
(14) Luc Boltanski et Laurent Thévenot, De la justification, Paris, Gallimard, 1991.

(15) Marie-France Marquès, "Charitéspectacle et travail social”, in Marquès et Walter, [dir.], Perspectives du travail social, Paris, ESF, 1988, p. 61.

(16) Comme le précise encore Marie-France Marquès (op. cit., p. 62), " une "Charité partie" a eu lieu durant cinq heures dans la nuit du 16 au 17 décembre. Cette coproduction d'Antenne 2 et de RTL était organisée avec le concours des salles de rédaction de la presse de certaines régions. En effet, avant l'émission, huit demeures avaient déjà été offertes à la fondation par des collectivités ou des particuliers. Lors du show télévisé, ces demeures étaient visualisées pour

\subsection{Les entreprises et impératifs de l'esthétisation}

Si les différents équipements évoqués supra participent tous, à des degrés divers, de l'esthétisation d'une souffrance, ils peuvent ne pas être suffisants pour nourrir les croyances évaluatives et stabiliser les définitions sociales de la souffrance et des réponses qu'il convient de donner. Les différents actants ne parviennent d'ailleurs pas à éviter certaines critiques, dont les plus courantes sont le voyeurisme ou encore la critique d'une justice spectacle, etc. Aussi, dans cette section, allons-nous nous écarter du cas de Johnny et mettre au jour deux autres équipements qui complètent et stabilisent aujourd'hui " ce qui ne doit plus se discuter" et proposent, en plus, des réponses isomorphes à celles des conduites et pratiques jusqu'ici labellisées du champ professionnel du social.

\subsubsection{Le renom comme équipement}

Comme l'ont bien montré Luc Boltanski et Laurent Thévenot, ${ }^{(14)}$ la compétition pour la recherche de l'état le plus valorisant n'a de sens que si elle contribue à l'état des biens communs. Certains agents, depuis une position de renom - qui suppose aujourd'hui une forte valeur ajoutée médiatique - reconnaissent certains traumatismes sociaux et investissent durablement les dispositifs de lutte ou de soutien d'une cause et, aujourd'hui, plus largement, le champ de l'intervention sociale. Cette position leur confere automatiquement (mais non définitivement) une grandeur qui peut servir de vitrine, de dispositif d'action directe ou distanciée et de label.

Ainsi, dans les années quatre-vingt, l'un des premiers à investir le champ en se fondant sur son renom est Jean-Luc Lahaye, lorsqu'il crée, en octobre 1986, la fondation Cent familles. Celle-ci compte aujourd'hui cinq centres qui accueillent plus d'une centaine d'enfants de 4 à 13 ans. Marie-France Marquès signale que "Jean-Luc Lahaye a passé son enfance, partagé entre le centre de l'Aide sociale à l'enfance de DenfertRochereau, la vie avec sa mère dans un quartier déshérité de Paris et de multiples familles de placement. Devenu chanteur, il publie, en novembre 1985, un livre retraçant son enfance (1.200.000 exemplaires vendus). Il décide alors d'affecter une partie des droits d'auteur à la création, à 
Clichy, d'un centre d'accueil pour enfants sans toit inauguré par François Mitterrand. [...] La fondation a pour objectif aussi d'aider les familles à retrouver un logement, un salaire ; elle souhaite, en effet, que les liens parents-enfants soient maintenus et consolidés. Le directeur signale que plusieurs familles choisissent ce centre car il permet d'éviter les placements par mesure administrative $"{ }^{(15)}$

Ici, la grandeur est d'abord associée au passé ; elle a supposé des sacrifices, un prix à payer dans une situation donnée. Les agents ayant connu et/ou vécu une situation de souffrance ou de précarité avant d'accéder au renom ne peuvent que se consacrer à rétablir une relation sociale contribuant au bien commun. Ces engagements doivent dès lors permettre sinon une transformation tout du moins une évolution pour répondre à des situations et effets contextuels (dans le cas de Cent familles, humaniser la DDASS). Pour consolider cet état, interviennent aussi les médias et leur pouvoir déclencheur et fédérateur. ${ }^{(16)}$

Autre illustration de cet engagement, l'association Perce-neige créée par le comédien Lino Ventura et son épouse pour répondre aux problèmes de prise en charge institutionnelle des personnes handicapées mentales. Le couple avait un enfant handicapé. Les six maisons Perceneige réparties en France offrent un nombre volontairement limité de places pour conserver un caractère familial et faciliter l'intégration ; elles bénéficient des agréments et financements classiques et d'un encadrement éducatif spécialisé. On trouve là la panoplie classique de ce type de prise en charge, à savoir des foyers d'hébergement, de vie, médicalisés, à double tarification, maison d'accueil spécialisée. Six autres maisons sont en projet à l'horizon 2000-2001.

Après le chanteur, le comédien, vient le sportif : Yannick et Marie-Claire Noah et leurs Enfants de la terre. Cette association créée en 1988 et déclarée de bienfaisance recueille des enfants en difficulté dans des structures d'accueil d'urgence baptisées les " maisons tendresse ». Ces maisons sont situées en Normandie, dans les Landes, dans les Pyrénées et en Bretagne. Cette association est particulièrement intéressante par les partenariats qu'elle génère et les équipements qu'elle mobilise pour assurer son fonctionnement. Elle organise le trophée des Enfants de la que les auditeurs puissent ainsi, par leurs dons, contribuer à

l'aménagement, à la rénovation ou au fonctionnement du centre d'accueil de leur région. De plus, les chefs d'entreprises et personnalités qui apportaient leurs aides recevaient en contrepartie un droit à la parole sur le plateau d'Antenne 2. Vedettes du spectacle, hommes politiques, PGG de nombreuses entreprises du bâtiment, fabricants de jouets ou de vêtements d'enfants pouvaient se rappeler au souvenir de 23 millions de spectateurs et démontrer leur "générosité". À la fin de l'année, les organisateurs annonçaient une collecte de 30 millions de francs". 
(17) Gilles Lipovetsky, Le crépuscule du devoir. L'éthique indolore des nouveaux temps démocratiques, Paris, Gallimard, 1992, p. 115.

(18) Il faut préciser que ces porte-parole qui ne sont pas exclusivement des stars sont en fait la première partie visible du dispositif de mise en scène d'un engagement dans le social, le premier plan de la vitrine des bonnes professionnalités. Il n'est plus rare de voir des émissions ou documentaires mettre en scène terre sous la forme d'une grande soirée tennis-concert retransmise sur Paris Première ou s'associe avec Vittel pour une opération de soutien. Opération dans laquelle la société verse $500.000 \mathrm{~F}$ destinés à faire vivre une de ces maisons pendant un an et s'engage à verser en plus $0,10 \mathrm{~F}$ pour chaque code barre envoyé par l'acheteur du produit. Par son intermédiaire on pouvait également acquérir un disque compact ou un teeshirt inédit de l'association. À la fin de cette opération, le relais est pris par Pic-Nik Break (biscuits et pâte à tartiner aux noisettes) qui s'engage à lui reverser pendant quatorze mois $0,50 \mathrm{~F}$ par paquet acheté. Dans ce dernier exemple, le renom est surtout fondé sur l'activité sportive qui, malgré toutes les dérives économico-médiatiques, est encore perçue, dans de nombreuses manifestations de solidarité, comme « une école de moralité cultivant le goût de la lutte, le sens de l'effort, la solidarité, le désintéressement. " ${ }^{(17)}$

\subsubsection{Les garants de légitimité}

Dans les exemples cités, le renom permet de mettre en équivalence l'authenticité et la qualité des conduites et pratiques et la réalité d'une souffrance éprouvée par des agents dont on connaît (bien) la grandeur et chez qui on ne suspecte aucune petitesse. Mieux même, ils ont déjà fait la preuve d'une compétence et de réussites et ont su résister à différentes épreuves. De facto, ils peuvent devenir fondateurs, "enrôleurs" ou porteparole de multiples prises de position (sur la réalité d'une souffrance sociale) et/ou d'interventions (pour y remédier). Si ces dernières s'inscrivent dans la durée, elles seront progressivement intégrées dans le déjà-là et fonctionneront avec des contraintes et logiques similaires aux autres organismes d'intervention sociale. D'où l'importance de ne pas se limiter à une seule grandeur et de stabiliser fréquemment ces constructions en les garantissant d'une certaine légitimité et de nombreuses alliances.

Ainsi l'appel ou le recours à des "garants de légitimité" est-il maintenant devenu un lieu commun auprès de très nombreux organismes d'intervention sociale. Pour certains, ils forment même un équipement essentiel pour éprouver l'utilité de leur existence eu égard aux souffrances auxquelles ils prétendent répondre. Ces garants sont invoqués et 
mobilisés dans une double perspective déclarée de popularisation et de soutien de la cause. Mais, là encore, ils ne restent qu'un soutien parmi d'autres tant qu'ils ne sont pas convoqués et associés à une exploitation des conduites et pratiques dans les médias et/ou dans des lieux d'exposition ou dans des événements.

Bien qu'équipement d'esthétisation aujourd'hui indispensable, la présence ou l'absence de garants de légitimité est très hétérogène selon les causes ou les événements. Utiliser sa notoriété, son parcours de vie suppose des choix et des accords sur la validité et l'authenticité de la cause. ${ }^{(18)}$ On le voit bien dans le cas des garants de légitimité évoqués supra, ce choix est une opération fort complexe, puisqu'elle associe des sentiments, des représentations, des éléments intériorisés participant du processus de socialisation des agents. Bref, l'alliance n'est, dans ce cas, jamais pure... coïncidence. Force est pourtant de constater que de plus en plus de "vedettes" s'investissent pour aider, participer, convaincre, soutenir ou mener la lutte contre les traumatismes sociaux du moment. Tous n'y ont pas une antériorité particulière avant de faire "cause commune". Par ailleurs, on a pu observer dans notre recherche que les causes les plus soutenues, à notre époque, sont "justement" celles concernant les enfants. À ceux qui dénoncent des opérations mercantiles indissociables aujourd'hui d'un bon "plan com.", les garants semblent démontrer par leurs actions que tout cela n'est plus incompatible. Pour tous il y a un intérêt, même si les actions ne sont pas intéressées. Dans les entretiens qu'ils accordent aux médias, leur engagement, c'est à l'évidence participer à un grand mouvement dont l'objet est le présent et l'avenir des enfants. Une cause concrète et qui se répercute aussi dans leur quotidien. Dans ce cas, l'engagement social ne se mesure pas simplement au temps passé ; on peut être garant occasionnellement, ponctuellement, mais toujours avec force. Leur présence permet aussi la transmission d'une rhétorique à la fois unitaire et omnibus dans les différents médias ou lieux de manifestation. Ils sont, en outre, "habitués" aux contraintes de temps (et donc de censure) qu'imposent les mises en scène médiatiques.

Pour illustrer ces propos, on peut se référer à l'association ELA qui lutte contre l'adénoleucodystrophie, maladie génétique rare, très handi- des histoires de vie, à

l'instar de celui qui retrace l'histoire d'une ancienne détenue chargée de mission par un ancien secrétaire d'État. On apprend que c'est au service des autres qu'elle s'est relevée, d'abord en aidant des filles en détresse, puis comme présidente d'une association lyonnaise pour des personnes sans-abri.

De même, les garants de légitimité peuvent également être des personnages fictifs s'engageant par le biais de leur créateur, comme certains dessinateurs de personnages de bandes dessinées. Ainsi Gaston Lagaffe soutient-il Greenpeace ou Amnesty International

(Tétérama, n² 2453, janvier 1997, p. 12). 
capante et actuellement sans grand espoir de guérison. Elle est reconnue d'utilité publique et multiplie les parrainages, notamment celui d'Emmanuelle Seignier (comédienne) et de Jean Galfione (champion olympique de perche). Dans l'émission de Canal +, Nulle part ailleurs (29/01/1998 entre 20h et 20h30), Guillaume Durand les reçoit pour l'annonce de deux grandes opérations : les kilomètres de l'espoir, où des enfants et adolescents des écoles et collèges organisent des courses et des cross et vont chercher des parrains (depuis octobre 1997, cette opération a déjà permis de récolter près de $800.000 \mathrm{~F}$ ) ; un bus itinérant dans Paris, qui "vend" des kilomètres en vue de subventionner une opération de recherche. Auparavant, les deux parrains expliquent l'origine et la nature de leur engagement pour cette cause. Pour la comédienne, tout est parti d'une rencontre avec une famille et leur garçon de huit ans atteint de cette affection ; pour elle « tout d'un coup, c'était concret ». Une rencontre qui agit comme événement transfigurateur (presque comme une révélation).

Autre exemple, celui de l'association Rêves, qui a pour vocation de réaliser les rêves d'enfants malades dont le pronostic est très réservé ; entre autres, permettre aux enfants de rencontrer une personnalité du monde artistique, sportif, culturel ou de la télévision, mais aussi découvrir un site ou parc de loisirs du monde animal. Elle a pour marraine la chanteuse et comédienne Vanessa Paradis (le parrain est Patrick Chêne, journaliste à France 2). Elle s'est entourée, lors d'un concert public, de plusieurs personnes de l'association; l'objectif de cette démarche était " que toute la médiatisation se fasse sur l'association, et pas sur nos petites personnes »; l'aide qu'elle apporte est, entre autres, de faciliter les démarches de l'association en direction de son métier.

On peut facilement multiplier les exemples. La comédienne Mathilda May s'occupe de l'association Chrysalide venant en aide aux enfants physiquement et psychologiquement maltraités et séparés de leurs parents par décision de justice. Cette association propose des placements en famille d'accueil et l'organisation d'un suivi thérapeutique jusqu'à leur majorité. Pour Mathilda May, d'après Téléstar ( $n^{\circ} 1054$, décembre 1996, p. 22-23), c'est sûrement le fait d'être mère qui l'a poussée à s'occuper de cette association. L'idée même d'un enfant qui souffre lui 
est insupportable. Paul Newman vient en aide à des associations au profit de familles sans ressources ou d'enfants atteints de maladies graves, ou encore crée une fondation pour venir en aide aux jeunes toxicomanes ou alcooliques qui fonctionne grâce à des dons, mais aussi grâce à des fonds provenant de la vente de produits alimentaires auxquels l'acteur a donné son nom. Karen Mulder défile gratuitement pour la Croix-Rouge et rêve de créer une fondation pour venir en aide aux enfants malheureux. Claudia Schiffer se consacre aux enfants malades et s'engage dans la lutte contre le cancer du sein. Il faut aussi évoquer ici les poupées Claudia Schiffer, Karen Mulder, Naomi Campbell vendues au profit de la CroixRouge (3 F par poupée sur $125 \mathrm{~F}$ ) ; la présentation du produit s'est faite dans l'émission du dimanche de Jean-Luc Delarue (France 2, le 21 avril 1996). Interrogée par un télémagazine, Karen Mulder déclare : "C’est une façon de penser et de faire penser aux enfants qui n'ont pas la chance de posséder un tel jouet. Quand, dans la vie, on a de la chance, on n'a pas le droit d'être radin et d'oublier ceux qui souffrent. "

Pour résumer le propos, on peut observer que les définitions des souffrances sociales, comme les façons d'y remédier ou d'y intervenir, visiblement sont aujourd'hui multiples et diversement équipées. On peut s'attaquer aux problèmes des banlieues par des dispositifs touristiques, culturels ou artistiques ; aligner les témoignages et les remèdes à la maltraitance autour d'un procès ou dans des psy show ; monter des opérations "séduction", "recueil de dons" ou "coup de poing" pour faire l'événement, opérations qui tendent, pour reprendre une formule de Jean-Yves Trépos, à laisser les bénéficiaires groggy après le départ des vedettes ; inventer des récompenses symboliques ou des gadgets humanistes, initier des pratiques cérémonielles pour consacrer des interventions et promouvoir les professionnalités... ${ }^{(19)}$ À côté de certaines pratiques, les campagnes humanitaro-médiatiques du début des années quatre-vingt sur les problèmes du tiers-monde, sans disparaître, appartiennent déjà à l'histoire.

Mais le plus important sans doute est que la construction des "bonnes définitions" d'une souffrance ou du malheur d'autrui passe aujourd'hui par des traductions et des choix. Ces traductions et choix par les équipements qu'ils mobilisent et dont on connaît la valeur ajou-
(19) Vincent Meyer,

"Téléthon, le oui mais de certains professionnels du travail social”, in Walter, [dir], Le téléthon. Scènes, intérêts, éthique, Paris, L'Harmattan, 1998, p. 141-159. 
(20) Jacques Lagroye,

"La production de la solidarité", in Produire les solidarités. La part des associations, document de la Mission recherche du ministère de l'Emploi et de la Solidarité, avec la collaboration de la Fondation de France, 1997, p. 14.

(21) Jacques Ion, La fin des militants?, Paris, éd. de l'Atelier, 1997, p. 81.

(22) Voir Jacques Walter, "L'entreprise saisie par le social et l'humanitaire.

Contribution à l'étude de la reconnaissance d'un monde professionnel”, Recherches en communication, $\mathrm{n}^{\circ}$ 6, 1997 ; et Le mécénat de solidarité. Communication, frontières et mondes professionnels, mémoire d'habilitation à diriger des recherches, université de Metz, 1997. tée médiatique - entraînent les non-souffrants dans un nouveau " militantisme de cause ", selon l'expression de Jacques Lagroye, ${ }^{(20)}$ ou dans des « engagements post-it », pour reprendre une expression de Jacques Ion. ${ }^{(21)}$ Ainsi, on choisit de soutenir telle cause et pas une autre, de favoriser telle ou telle prise de conscience, d'expérimenter ou non (et pendant un temps) tel ou tel dispositif, de donner à voir tel ou tel aspect de l'engagement tant du côté du grand public, des bénévoles que des professionnels. Le développement important du mécénat de solidarité va également dans ce sens. ${ }^{(2)}$ Mais, comme cela a déjà été évoqué supra, ces choix servent aussi à distinguer, à singulariser, à préférer ou à prendre de la distance par rapport aux souffrances ou malheurs d'autrui. Tout se passe comme si, avec ces "bonnes définitions", se réalisait la conjonction de différentes possibilités de connaître et de différentes possibilités d'agir, pour reprendre une formule de Luc Boltanski. ${ }^{(23)}$ De même, un seul engagement peut valoir - par une médiatisation consciente et méthodique - pour plusieurs (voire plusieurs milliers).

\section{La mise "en cause" des professionnalités}

Dans nos travaux, nous avons constaté un lien entre l'esthétisation de la souffrance et l'esthétisation d'une professionnalité. Ce constat doit maintenant être approfondi. On l'a vu, l'affaire du petit Johnny a conduit un certain nombre "d'interprètes autorisés" à se poser de "nouvelles" questions sur les rôles et compétences avérés de différents professionnels. Au premier plan, ceux des services sociaux; dans une moindre mesure, ceux de la justice et des médias.

Pour les premiers - et plus spécifiquement les travailleurs sociaux qui ont licence et mandat (au sens de Hughes ${ }^{(24)}$ ) pour prévenir et/ou répondre à cette forme de souffrance -, ce type d'affaire devient une véritable épreuve de professionnalité. Épreuve qui s'inscrit aussi dans un contexte plus global : d'incertitudes sur le "comment faire du social aujourd'hui" ; de transformations des professions labellisées et des modes d'exercice "historiques". ${ }^{(25)}$ Bref, lorsque la souffrance attire l'attention des médias, les nouvelles définitions qu'ils construisent sont aussi lourdes d'effets de représentation et d'épreuves de professionnalité qu'il convient 
de ne plus ignorer. Cette "mise à l'épreuve" ne concerne d'ailleurs pas uniquement les professions du social ; pour emprunter une expression de Jacques Walter, ${ }^{(26)}$ celles de la justice n'ont pas encore réussi, elles non plus, « à passer de l'image subie à l'image voulue ".

\subsection{Le nécessaire travail médiatique du social}

Parmi toutes les questions que pose le cas du petit Johnny, il en est une qui est revenue avec force dans l'ensemble de l'épisode juridico-médiatique : comment cette maltraitance a-t-elle échappé aux services sociaux ? En effet, dans la mise au jour de ce type d'affaire, tout se passe comme si les médias validaient immédiatement l'échec et une remise en cause des professionnalités, les professionnels étant dès lors sommés d'apporter leurs preuves, mais aussi de revaloriser une image ternie et subie.

\subsubsection{Stigmatisation ou dévalorisation de la professionnalité des travailleurs sociaux?}

Il est une difficulté récurrente dans le métier de travailleur social, celle de mesurer et/ou d'évaluer les "dangers" encourus par les différents usagers ou ayants droit, d'autant que ceux-ci sont devenus, pour reprendre une belle formule de Jacques Ion, "de moins en moins prédéfinissables". C'est le cas notamment lors des signalements de maltraitance d'enfants. Il semble qu'il reste très difficile de diagnostiquer l'enfant en danger au sein de sa famille ou, plus exactement, de mesurer la réalité du danger encouru par l'enfant et donc d'évaluer le moment "opportun" de signalement et d'intervention. Là encore, les multiples esthétisations produites par différents équipements coconstruisent des définitions et des formes d'action que ne maitrisent pas forcément les travailleurs sociaux.

L'un de ces équipements est notamment la mise en place d'un service national d'accueil téléphonique avec un numéro simplifié (Allô Enfance maltraitée : 119) ; l'appel est gratuit et n'apparait pas sur les factures de téléphone. Au bout du fil, quarante professionnels de l'enfance répondent $24 \mathrm{~h} / 24$ aux demandes d'information et de conseil concernant les situations de mineurs maltraités ou présumés l'être ; plus de 200.000 appels en $1995 .{ }^{(27)}$ Cette illustration donne l'occasion de s'arrê-
(23) Luc Boltansky, op. cit., 1993, p. 22.

(24) Everett Hughes, Le regard sociologique, Paris, EHESS, 1996.

(25) Sur ce point, voir : Jean-Noël Chopart, [dir.], Les mutations du travail social. Dynamiques d'un champ professionnel, Paris, Dunod, collection "Action sociale", 2000.

(26) Jacques Walter, Directeur de communication : les avatars d'un modèle professionnel, Paris, L'Harmattan, 1995, p. 74.

(27) Vies de famille, trimestriel de la CAF de Moselle, n 1, mai 1997. 
(28) Voir notamment les travaux d'Éveline Lehnisch, "Les services d'aide par téléphone", in Marquès et Walter, [dir.], Perspectives $d u$ travail social, Paris, ESF, p. 51-56.

(29) Notamment du fait : de la gratuité du service et de l'appel téléphonique; de la rapidité de la réponse sans déplacement ; que le téléphone peut pallier l'impossibilité ou la difficulté d'exprimer sa détresse dans un face-à-face ; enfin, de l'anonymat de l'entretien qui peut, dans certaines situations, favoriser l'analyse approfondie de la situation et préparer une rencontre ou une démarche avec un autre intervenant.

(30) L'enfance maltraitée a été grande cause nationale 1997 avec pour slogan « Si tout le monde bouge, ça bougera ». Il faut préciser ici que la forme "grande cause nationale » est renouvelée chaque année et bénéficie d'un agrément par le Premier ministre. Cette campagne sur la protection de l'enfance maltraitée avait notamment pour objectif de combattre les violences sexuelles. Plusieurs moyens ont été mis en œuvre, avec notamment un projet de loi pour que les coupables soient soumis à une peine complémentaire de suivi médicosocial, une amélioration des procédures policières et judiciaires d'accompagnement des enfants victimes, la prise en charge thérapeutique des enfants et une campagne de sensibilisation de tous les professionnels impliqués, ainsi que des parents, des jeunes et des enfants.

(31) J. Lazar, Sociologie de la communication ter brièvement sur l'accroissement et le développement, au milieu des années quatre-vingt, dans les milieux associatifs et institutionnels, des numéros verts et des services d'aide par l'objet technique téléphone. Pour d'aucuns, ${ }^{(28)}$ le réseau d'aide par téléphone remplit une fonction sociale réelle en complémentarité d'autres formes d'aide et de soutien. ${ }^{(29)}$ Par ailleurs, le téléphone illustre bien ce que peut être l'évolution de l'objet technique au service d'autrui et/ou à vocation solidaire, permettant aussi une réduction du coût de l'intervention humaine. Dans la concurrence actuelle entre les opérateurs de télécommunication, où tout va se jouer sur le service offert, on peut raisonnablement penser que l'un d'entre eux pourrait se découvrir une fibre sociale et trouver quelque intérêt au développement de ces interventions à distance ou distanciées.

Mais il faut bien comprendre que tout ceci prend avec d'autant plus de force que l'enfance maltraitée est devenue une cause indiscutable, ${ }^{(30)}$ que les médias - en dépassant leur fonction principale de "diffuser l'information et la connaissance $"{ }^{(31)}$ - redistribuent, nuancent ou enrichissent les connaissances établies sur les phénomènes de maltraitance. Mais surtout, pour appuyer le propos de Patrick Champagne, parce que "les médias font désormais partie intégrante de la réalité ou si l'on préfere produisent des effets de réalité en créant une vision médiatique de la réalité qui contribue à créer la réalité qu'elle prétend décrire. " ${ }^{(32)}$

Ainsi, pour ne prendre que cet exemple, la télévision met-elle de plus en plus en scène des pans particuliers de diverses professions et principalement, dans ce qu'on a pu observer, celles du secteur des 
biens et services. On pense bien sûr de suite à l'équipement reality showe. ${ }^{(33)}$ Ces magazines de télévision - autant suivis que décriés - ont mis en scène, plus particulièrement depuis le début des années quatre-vingt-dix, à la fois des situations et questions sociales, des professions, des individus ordinaires et des experts. Une scène occupée, pour Jean-Yves Trépos, « par la rencontre conflictuelle d'experts reconnus dans un champ professionnel et de contre-experts, en un débat qui ne pourrait avoir lieu nulle part ailleurs. ${ }^{(34)}$ Selon Dominique Mehl, ces magazines participent d'un interventionnisme télévisuel et d'une télévision de l'intimité qui mêlent « deux inspirations conjointes : l'irruption de la parole profane sur la scène publique d'un côté, la valorisation du témoignage privé de l'autre. » ${ }^{(35)}$ Cette télévision du témoignage peut être un soutien à la consolidation des rhétoriques de légitimation des professionnalités ou au contraire de dénonciation, dans la mesure où ces magazines "s'inscrivent dans un mouvement plus large de suspicion à l'encontre des savoirs officiels et académiques, [...] de valorisation de l'expérience profane ".

Signalons, enfin, que si tous ces équipements et esthétisations participent autant d'une stigmatisation et/ou dévalorisation de la professionnalité des travailleurs sociaux, c'est que ces derniers ne font pas grand chose pour y "faire front" et/ou pour ne pas les subir. Ceci est patent dans le cas de Johnny. Aujourd'hui, la manifestation d'une compétence dans l'espace public passe nécessairement par des esthétisations (appelées dans ce cas des protocoles) qui se caractérisent par l'obligation de toujours lier le "faire" et/ou le "faire faire" au "pour quoi faire" et d'y inclure le "comment faire savoir pourquoi on fait". Autrement dit, la capacité à communiquer ou à communiquer mieux commence à s'imposer à toutes les rhétoriques professionnelles.

\subsubsection{Se plier à l'impératif communicationnel}

Au final, la question centrale pourrait être : comment une médiatisation réfléchie et organisée permet-elle d'éviter certaines épreuves ${ }^{(36)}$ et d'apporter des preuves ? Du côté des professions sociales, il est largement temps de mettre en place une politique de communication en direction du "grand public" et de spectateurs professionnels (financeurs, déci- de masse, Paris, Armand

Colin, 1991, p. 146.

(32) Patrick

Champagne, op. cit., 1993, p. 74.

(33) L'exemple archétypal du moment étant le magazine Ça se discute de Jean-Luc

Delarue, sur France 2.

(34) Jean-Yves Trépos, Sociologie de la compétence professionnelle, Nancy, Presses universitaires de Nancy, 1992, p. 56.

(35) Dominique Mehl, La télévision de l'intimité, Paris, Seuil, 1996, p. 8.

(36) Voire certains pièges, pour reprendre l'expression de la psychologue "piégée" au moment du procès (voir point 1). 
(37) Everett Hughes, op. cit., p. 119.

(38) Qui ne se limite pas à ce que d'aucuns appellent la "communication sociale".

(39) On est presque tenté de dire qu'ils sont toujours engagés du simple fait qu'ils les décrivent, donnent leur version des faits ou les ignorent. On rejoint ici Dominique Mehl, pour qui « la télévision, deurs, parents, etc.). Ce passage à la communication vise d'abord à donner une image pensée comme positive et à se (re)présenter comme utile voire indispensable, notamment pour ne plus dépendre de la seule "vision médiatique". Ensuite, il doit permettre de distinguer des interventions aujourd'hui relativement similaires (fonctions généralistes d'accueil, d'accompagnement, de suivi, etc.) et mettre l'accent sur certaines spécificités ou complémentarités (niveau de qualification, travail en équipe pluridisciplinaire, infrastructure, etc.) par rapport à d'autres professionnels et/ou bénévoles. Cet impératif communicationnel, qui se développe dans tous les champs professionnels, peut aussi demander le recrutement d'agents externes compétents en la matière, des "spécialistes en relations publiques [qui sont engagés] afin de réaliser ce travail délicat qui consiste à donner une image publique satisfaisante. " ${ }^{(37)} \mathrm{Ceci}$ est d'autant plus utile que les travailleurs sociaux ne sont pas encore préparés (a fortiori formés) aux exigences du discours informatif attendu par les médias ou de celui à produire pour les intéresser, si - et seulement si - le calendrier des événements médiatiques (ce que d'aucuns nomment l'actualité) l'autorise.

Force est donc d'admettre que, dans l'attente d'une professionnalisation établie de la communication d'intervention sociale, ${ }^{(38)}$ ce sont les médias qui contrôlent l'accès aux différents moyens de diffusion. Les travailleurs sociaux doivent donc apprendre à communiquer sur leur professionnalité et à collaborer avec les médias. Ceci est d'autant plus important que, par ailleurs, ces derniers peuvent être ou non engagés dans différentes conduites et pratiques isomorphes à celles des travailleurs sociaux ${ }^{(39)}$ et sont parfois simultanément promoteurs ou producteurs de nouvelles formes d'intervention sociale et vecteurs de la mise en scène ou de la manifestation de formes plus anciennes dans l'espace public ; c'est ce que nous avons appelé le travail social des médias. Signalons toutefois que certains travailleurs sociaux refusent d'entrer dans l'arène, de manifester leurs compétences dans l'espace public, préférant et légitimant (notamment sous couvert de secret professionnel) un fonctionnement pro domo, pour eux nullement contradictoire avec l'évolution d'une société où tout se communique. 


\subsection{Justice et médias}

Il ne parait pas exagéré d'avancer que cet impératif communicationnel et/ou la collaboration avec les médias s'imposent aujourd'hui à tous les groupes professionnels. On voudrait ici évoquer brièvement certains résultats d'une enquête réalisée à l'occasion d'un colloque du Centre de recherche sur les médias intitulé "Télévision, justice et régulation". Cette enquête met en lumière les tensions complexes qui président à la construction des interactions, des mécanismes d'échange d'information et des stratégies d'investissement entre deux groupes professionnels : celui de la justice et celui des professionnels de la télévision. Ces interactions nous amènent aussi à considérer sous un angle différent ce que d'aucuns nomment une "déferlante ou une pression médiatique" lors d'un procès comme celui dont il a été question dans ce texte.

Dans sa dimension qualitative, l'enquête montre d'abord que ce sont deux mondes qui se sont longtemps pratiquement ignorés. On apprend ainsi que le judiciaire ne sait pas toujours comment travaille une télévision et vice versa. Elle confirme ensuite l'intérêt croissant des médias, et plus particulièrement de la télévision, pour l'institution et les affaires judiciaires. Cet intérêt semble pourtant être suscité en grande partie par les usagers eux-mêmes. Cette étude n'a, bien sûr, pas permis de mesurer dans cet intérêt ou engouement le degré de détermination suscité par les médias. Reste que la médiatisation télévisuelle des pratiques judiciaires parait, depuis plusieurs années, passionner les Français et que, de l'avis de certains professionnels de la télévision, ce sont les usagers qui poussent au développement de cette médiatisation.

Ainsi, pour les professionnels de la télévision, peut-on clairement parler de "stratégies d'investissement prioritaires " dans l'espace judiciaire et dans le champ professionnel de la justice. Ces stratégies sont nécessaires, voire incontournables, pour l'exercice même de leur profession ; parmi les plus importantes, on peut sérier : la recherche de contacts en vue d'obtenir des informations et/ou des images ; la course à l'audience et donc au scoop ; l'apprentissage de nouvelles compétences techniques et/ou linguistiques pour maîtriser le déroulement des procès et des procédures. Ces stratégies d'investissement prioritaires se conjuguent à leur 
capacité de mettre en scène de nombreuses réalités professionnelles et/ou sociales, et l'ensemble participe d'une forme particulière d'expertise participante que ne maitrise pas l'institution justice et qui peut, de manière circonstancielle, régler et/ou régir un certain nombre de relations et/ou de situations.

L'enquête montre encore que les professions judiciaires semblent se limiter, pour l'heure, à des mécanismes d'échange occasionnels et/ou circonstanciés, tout en restant dans une prudente expectative pour ce qui concerne la publicisation de leurs conduites et pratiques dans l'espace public. En d'autres termes, la communication judiciaire ne passe pas forcément par la communication télévisuelle (ou, plus largement, médiatique). L'avenir d'une transparence de l'actualité judiciaire, d'un débat judiciaire médiatisé et largement diffusé n'est pas pensé prioritairement en relation ou par des échanges formalisés avec les médias. La justice n'a pas encore besoin des médias pour combler un éventuel déficit de légitimité, des dysfonctionnements ou un manque de moyens, mais peut-être doit-elle avoir recours aux médias pour gagner en visibilité et ainsi faire mieux connaître son fonctionnement. Cet enjeu semble d'autant plus important que "l'américanisation" des représentations (par exemple, les formes de justice spectacle; mais aussi les émissions interactives, les téléfilms ou héros récurrents mettant en scène le fonctionnement de la justice), induite plus spécifiquement par la télévision, peut modifier l'image de la justice.

L'approche quantitative nous montre, en premier lieu, que les usagers questionnés accordent une place, une légitimité et des capacités d'influence importantes aux médias et surtout à la télévision. Si elle apparaît surtout comme outil central d'une information, certes sélectionnée en raison des impératifs d'audience, elle est également perçue comme pouvant servir d'outil d'investigation et d'élucidation pour certains problèmes sociaux et politiques contemporains. Cette compétence nouvelle peut d'ailleurs s'exercer sans licence ou mandat particulier (ou, au contraire, en les coconstruisant au fil des expériences télévisuelles) et surtout sans obligation de réussite dans sa volonté d'assurer telle ou telle fonction spécifique. En second lieu, elle met au jour le fait que, du 
côté des usagers, tout se passe comme si le débat public se centrait davantage sur les “crises" (40) de l'institution judiciaire. La multiplication des affaires et leur élucidation plus ou moins rapide entraînent dans l'opinion un véritable travail de représentation, que nous n'avons pas été en mesure de décrire et d'analyser finement dans cette enquête et qui semble créer un environnement hétérogène susceptible de forcer la réactualisation ou la transformation de certaines conduites et pratiques dans chacun des groupes professionnels.

\section{Conclusion}

Nos travaux de recherche ${ }^{(41)}$ ont permis d'observer que les rôles et fonctions d'information, de (re)présentation, de communication et de médiatisation prennent une place de plus en plus importante dans la définition des souffrances et traumatismes dits sociaux et, plus largement, dans la conception et l'élaboration des diverses formes de professionnalité.

Sur la définition des souffrances, on peut tout de suite objecter, avec raison sans doute, qu'elle peut toucher de manières diverses les agents, que l'on ne maîtrise pas les mécanismes de la réception, a fortiori leur influence sur les croyances évaluatives. Bref, que personne ne voit, ne croit et/ou ne reçoit de la même façon la souffrance d'autrui. L'accord est donc difficile à établir et à stabiliser. Ceci posé - et le traitement médiatique de l'affaire du petit Johnny en est une bonne illustration il y a des chances que le sens (ou l'essentiel) de la souffrance ne soit plus (inscrit) dans cette souffrance. Ce sens differe selon la réalité traitée, la nature des événements et des équipements mobilisés. Clairement, aujourd'hui, ce sont les équipements médiatiques qui permettent d'inscrire, de positionner et de visibiliser cette souffrance dans l'espace public, notamment parce qu'ils agissent à la fois comme informateurs, traducteurs, filtres et/ou écrans. Ainsi, autant dans le traitement médiatique du malaise des banlieues, les médias " peuvent ne pas prendre parti ou ne pas choquer des auditoires socialement très hétérogènes [et produisent] une présentation artificielle et neutralisante de tous les points de vue en présence ", ${ }^{(42)}$ autant dans celui d'une maladie génétique ou de
(40) Dysfonction-

nements, erreurs,

lenteurs, contrôle, manipulation, etc.

(41) Voir notamment : Vincent Meyer, “Les compétences sous l'œil expert des médias : les intervenants sociomédiatiques", Utinam, $\mathrm{n}^{\circ} 23$, Paris, L'Harmattan, 1997 ; Interventions sociales et médias entre professionnalisation et déprofessionnalisation, thèse pour le doctorat de sociologie, université de Metz, 1998 ;

"Intervention sociale et communication télévisuelle : exclusion ou complémentarité”, in Jacques Walter, [dir.], Télévision et exclusion, Paris, L'Harmattan, 2001.

(42) Patrick Champagne, op. cit., p. 68. 
l'enfance maltraitée, il faut produire par les faits et effets médiatisés de l'émotion authentique, provoquer et redistribuer l'indignation, la compassion et l'engagement et, in fine, les esthétiser de façon à ce que toute personne, face à cette souffrance, sans quitter sa réalité, sans nécessairement entrer dans une relation de face-à-face avec le souffrant, se montre affectée et déclenche l'action.

$\mathrm{Au}$ travers de cette recherche, on a également montré comment se manifestent dans l'espace public différents profils professionnels et comment les professionnels, les bénévoles, les décideurs (voire dans certains cas les usagers) sont mis - et/ou sont sommés de se mettre - en scène. En effet, aujourd'hui, peu de professions échappent à l'image que l'on se fait d'elles ; l'acte de faire voir ou d'être vu dans l'espace public, même s'il comporte des risques, devient un équipement indispensable d'un processus de professionnalisation. Il est d'autant plus indispensable pour des intervenants sociaux professionnels ou bénévoles qui revendiquent une utilité sociale et prétendent répondre aux souffrances et traumatismes sociaux du moment.

La place a manqué dans le présent article pour développer plus finement comment les médias, et principalement la télévision, participent aujourd'hui d'un processus de redéfinition et de coconstruction des professionnalités du social et, plus largement, à la constitution d'un champ que nous avons appelé "socio-médiatique". Ceci, à la fois sur sollicitation de différents intervenants sociaux saisis par l'impératif communicationnel (autrement dit, le travail médiatique du social), mais aussi de leur propre initiative, lorsqu'ils s'investissent dans le social ou créent eux-mêmes leurs interventions en s'emparant d'opportunités offertes par l'actualité, sans pour cela se couler dans une forme d'intervention labellisée ou définie $a$ priori (autrement dit, le travail social des médias).

Dans le socio-médiatique, nous sommes donc en présence d'un vaste ensemble de conduites et de pratiques qui continuent comme par le passé à s'accumuler, mais qui coexistent et s'interpénètrent beaucoup plus facilement et plus "im-média-tement". On a ainsi observé que, dans ce contexte mouvant, les montées en généralité et en humanité font rapidement cause commune. Bref, les volontés de médiatiser le social et 
de se servir des médias pour faire du social sont aujourd'hui multiples. Le vocabulaire comme la grammaire du socio-médiatique n'excluent $a$ priori aucune expression des problèmes sociaux et/ou conjugaison des réponses qu'on cherche ou qu'on a intérêt à y apporter. Surtout lorsque ces expressions croisent un développement technologique comme celui des équipements médiatiques, maintenant inscrit dans la durée, incluant et permettant un changement d'échelle, de temporalité, d'équipements et de territoires, et s'appuient sur lui. 